\title{
Proteogenomic approach to UTR peptides identification
}

Seunghyuk Choi, ${ }^{\#,+}$ Shinyeong Ju, ${ }^{\#,, \neq}$ Jinwon Lee, ${ }^{\#,+}$ Seungjin Na, ${ }^{\dagger}$ Cheolju Lee, $,{ }^{*},, \|, \perp$ and Eunok Paek ${ }^{*,+}$

${ }^{\dagger}$ Department of Computer Science and ${ }^{\ddagger}$ Department of Life Science and Research Institute for Natural Sciences, Hanyang University, Seoul 04763, Republic of Korea

${ }^{\S}$ Center for Theragnosis, Korea Institute of Science and Technology, Seoul 02792, Republic of Korea

"Division of Bio-Medical Science \& Technology, KIST School, Korea University of Science and Technology, Seoul 02792, Republic of Korea

${ }^{\perp}$ KHU-KIST, Kyung Hee University, Seoul 02447, Republic of Korea

"These authors contributed equally.

*E.P.: E-mail: eunokpaek@hanyang.ac.kr. Tel: +82 2-2220-2377.

*C.L.: E-mail: clee270@kist.re.kr. Tel: +82 2-958-6788.

\section{Table of Contents}

Cover

Description . S2

Comparing graphs of 29 pairs of the UTR peptides and the SIS peptides S3-16 


\section{Supporting Information S3.}

To present the evidence of the presence of the UTR peptides, we compare the spectral similarity between UTR peptides and SIS peptides. We used MS/MS spectra of UTR peptides and SIS peptides to compare, however, SIS peptides have isotopically modified in C-terminal residue to distinguish their mass from SIS peptides. Hence, y-ion series of fragmented ions will unmatch each other consequently. To resolve this, we intentionally transformed y-ions and precursor ions matched mass peaks in the spectra of the SIS peptides to the $\mathrm{m} / \mathrm{z}$ values without isotopes.

This document contains comparing graph of 29 pairs of the UTR peptides and the SIS peptides. Each page contains the sequence of the pair of peptides, and their spectrum similarity. We elucidated the graph using the function SpectrumSimilarity of OrgMassSpecR package with $0.01 \mathrm{~m} / \mathrm{z}$ tolerance (ver 0.5-3, URL: http://OrgMassSpec.github.io/). Each spectrum in the same peptide was compared using spectral contrast angle.

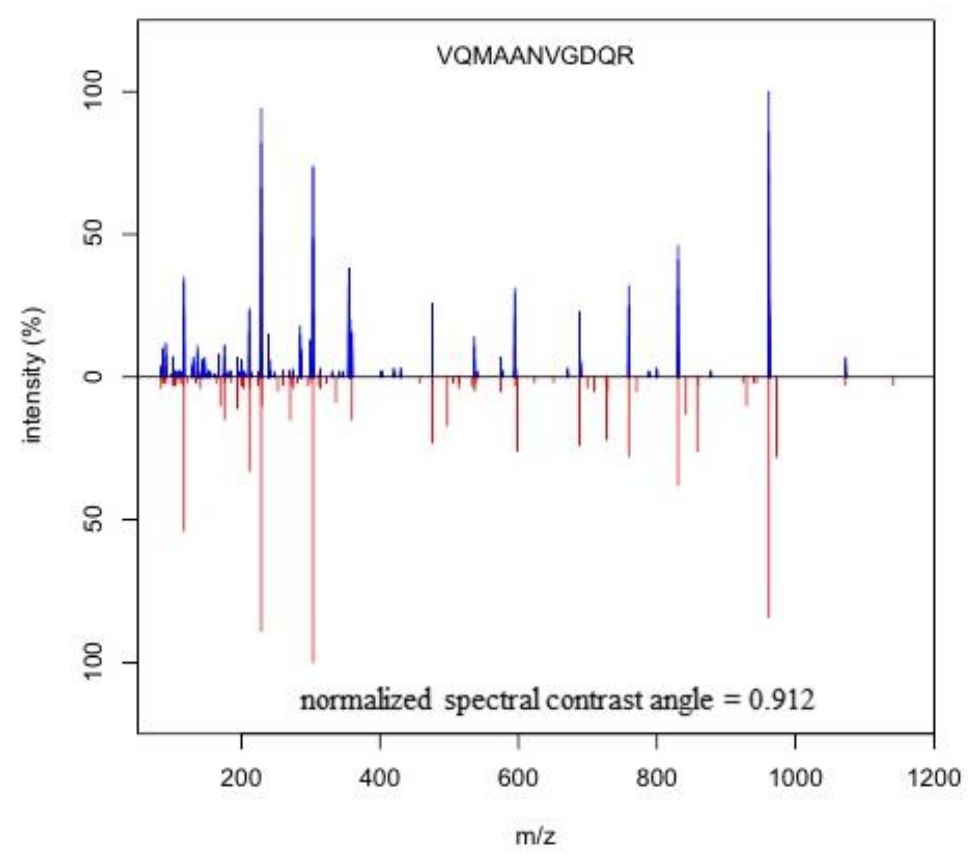



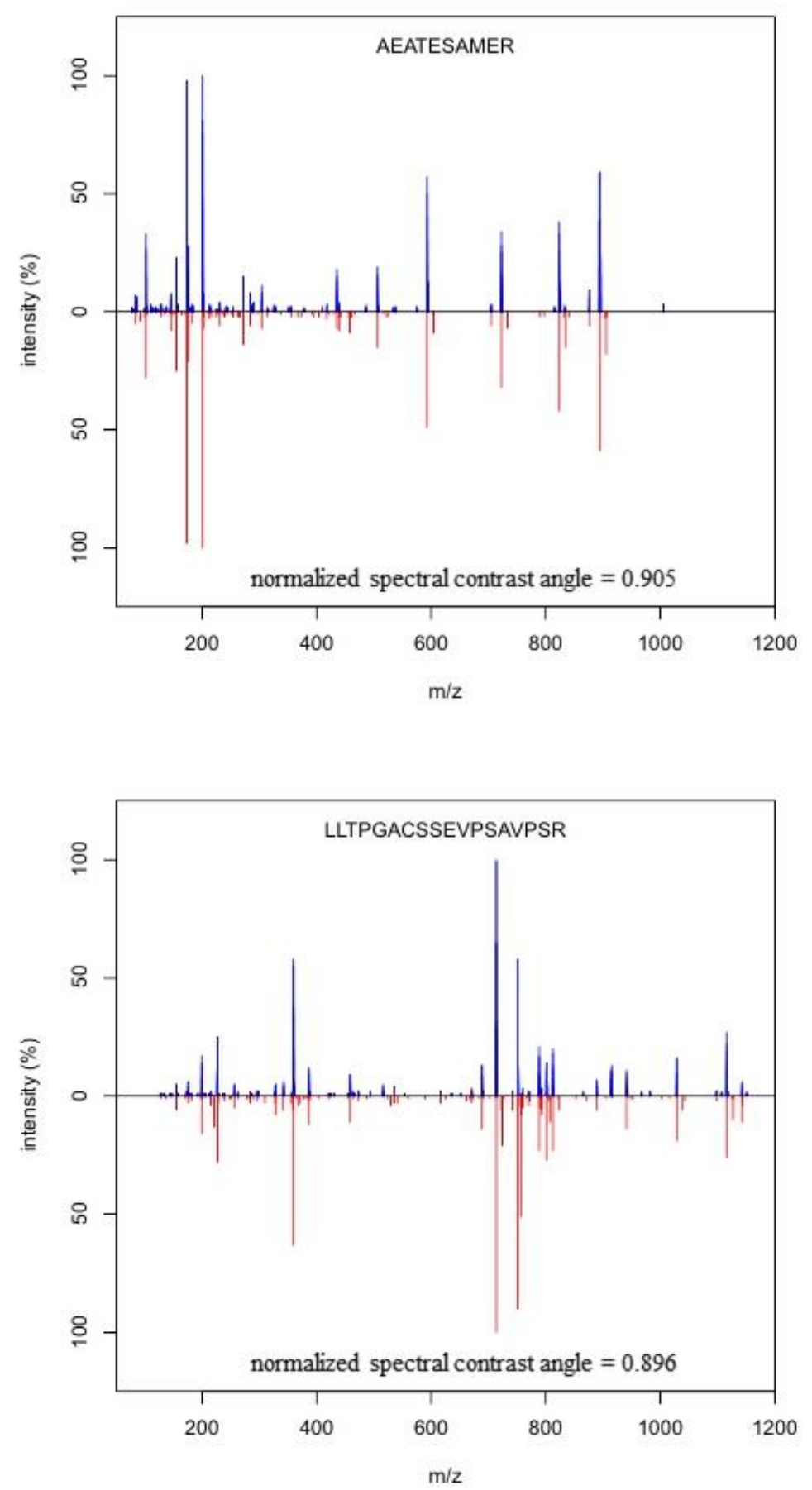

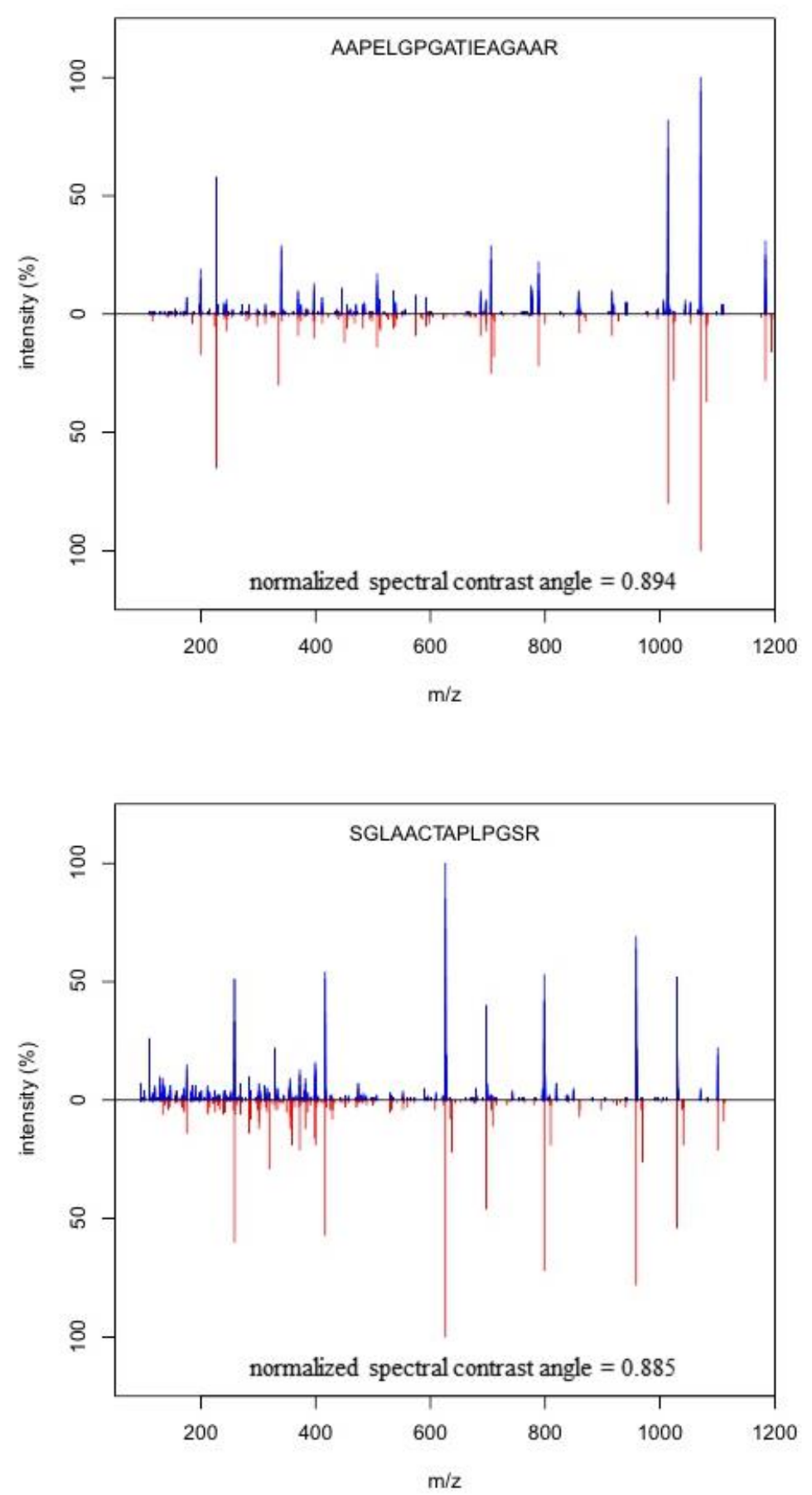

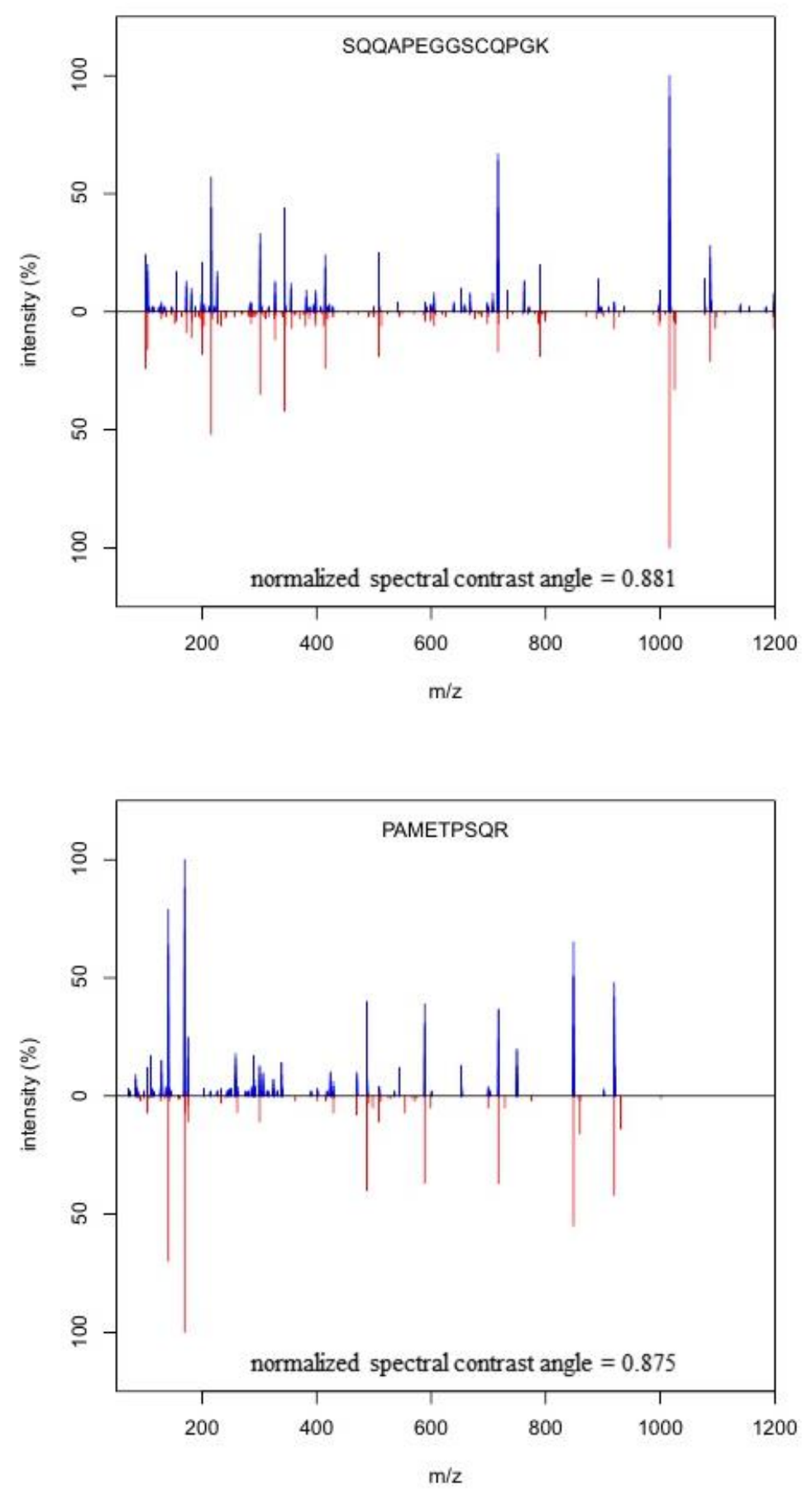

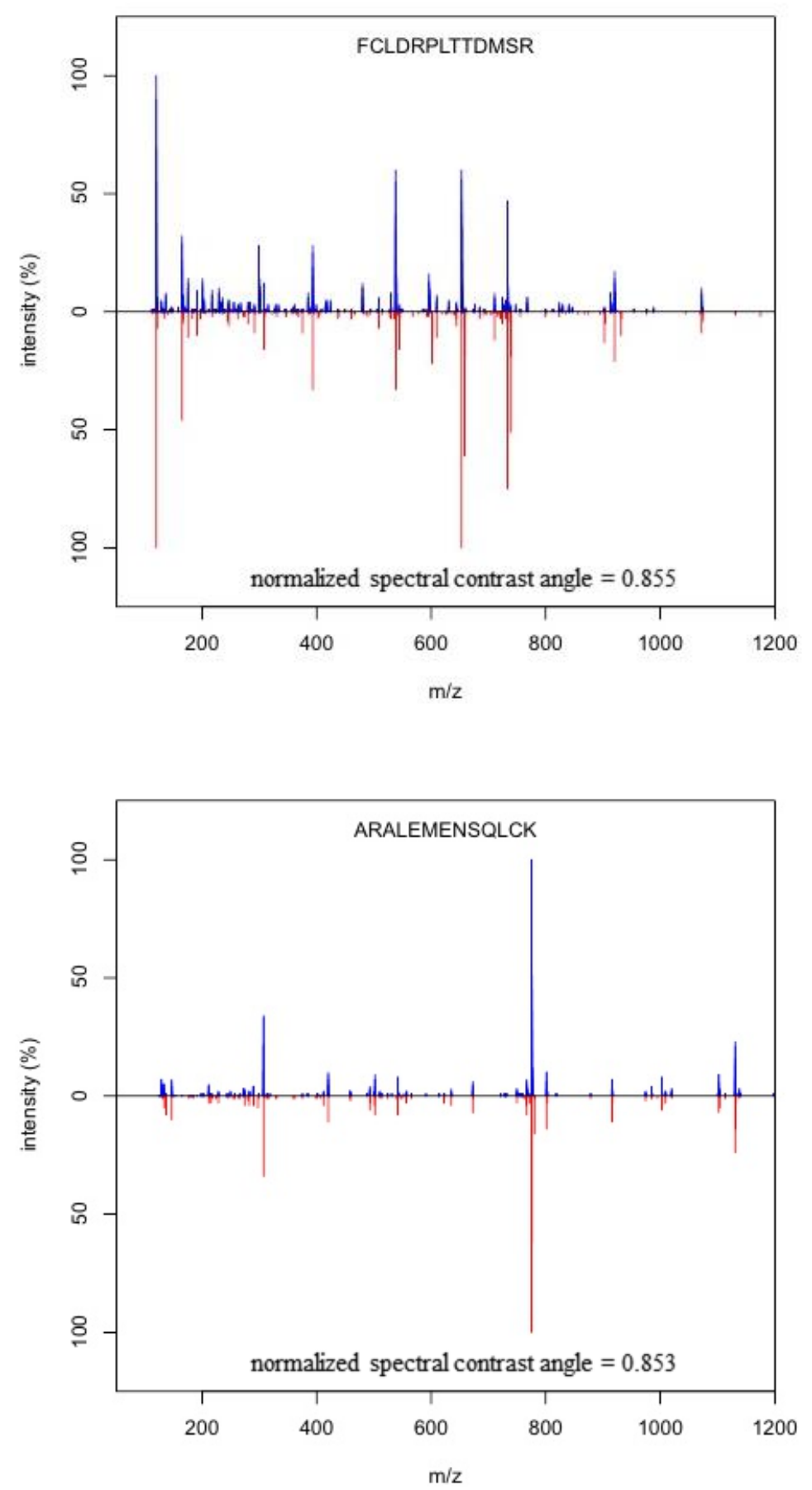

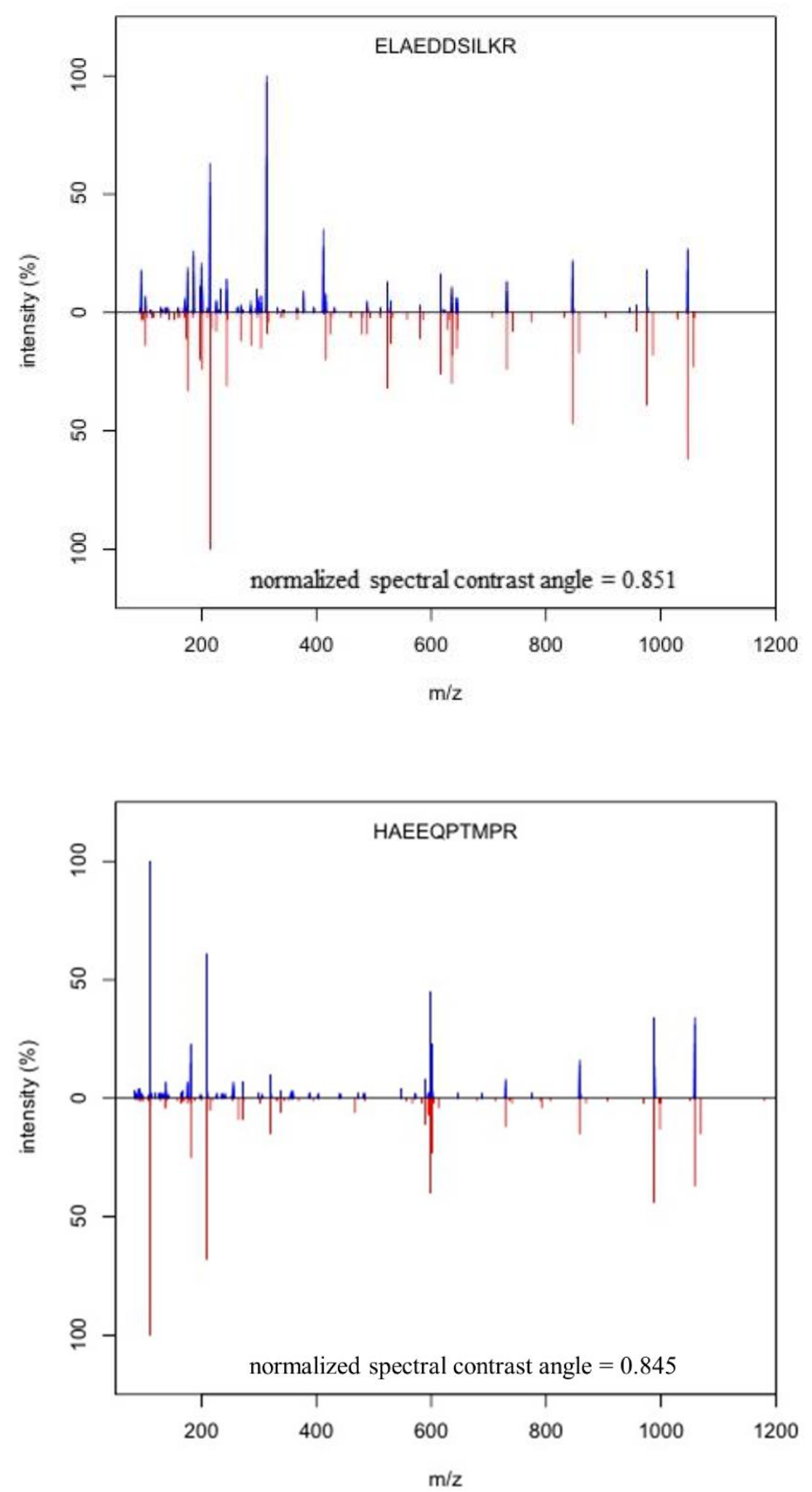

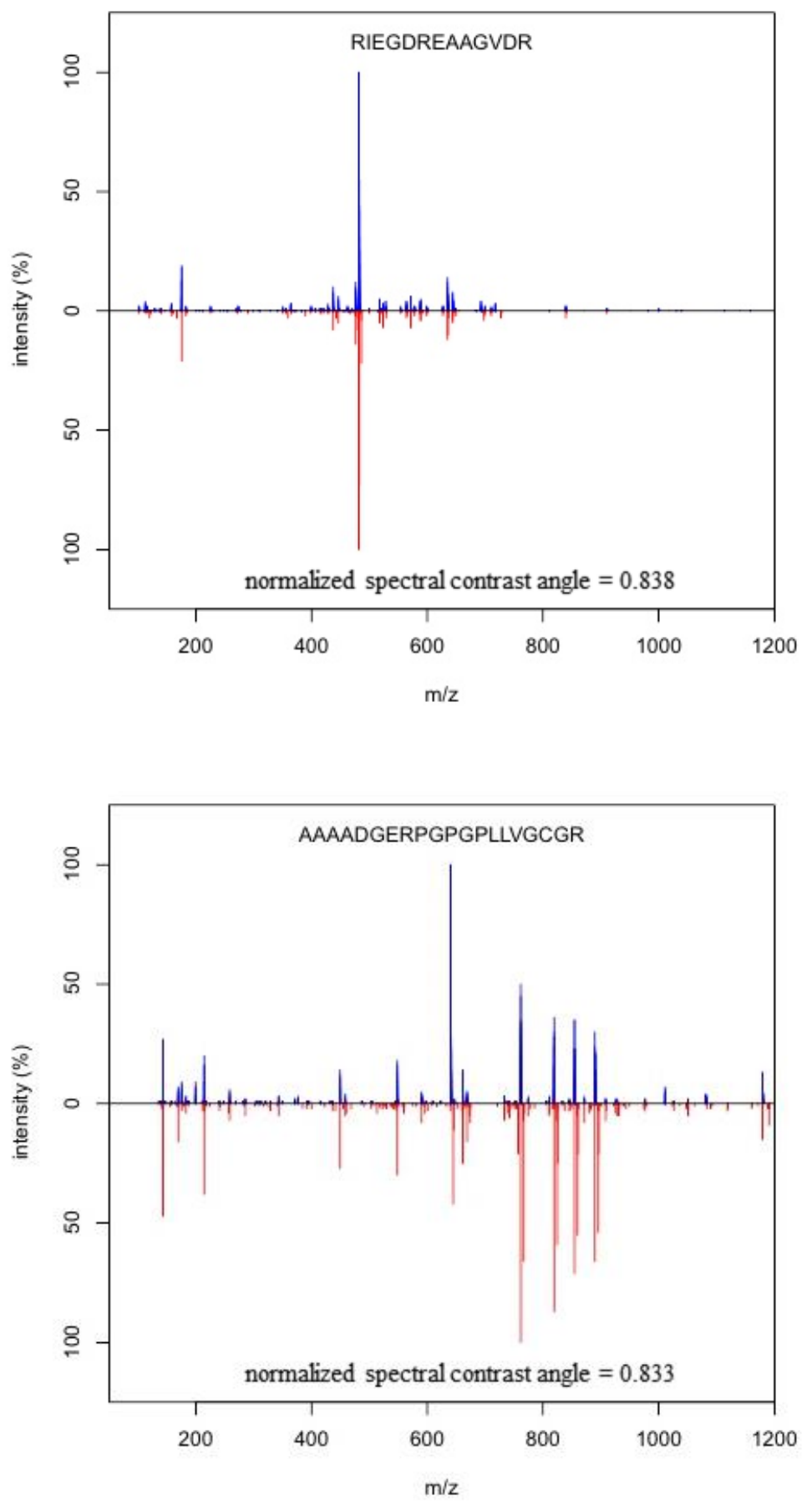

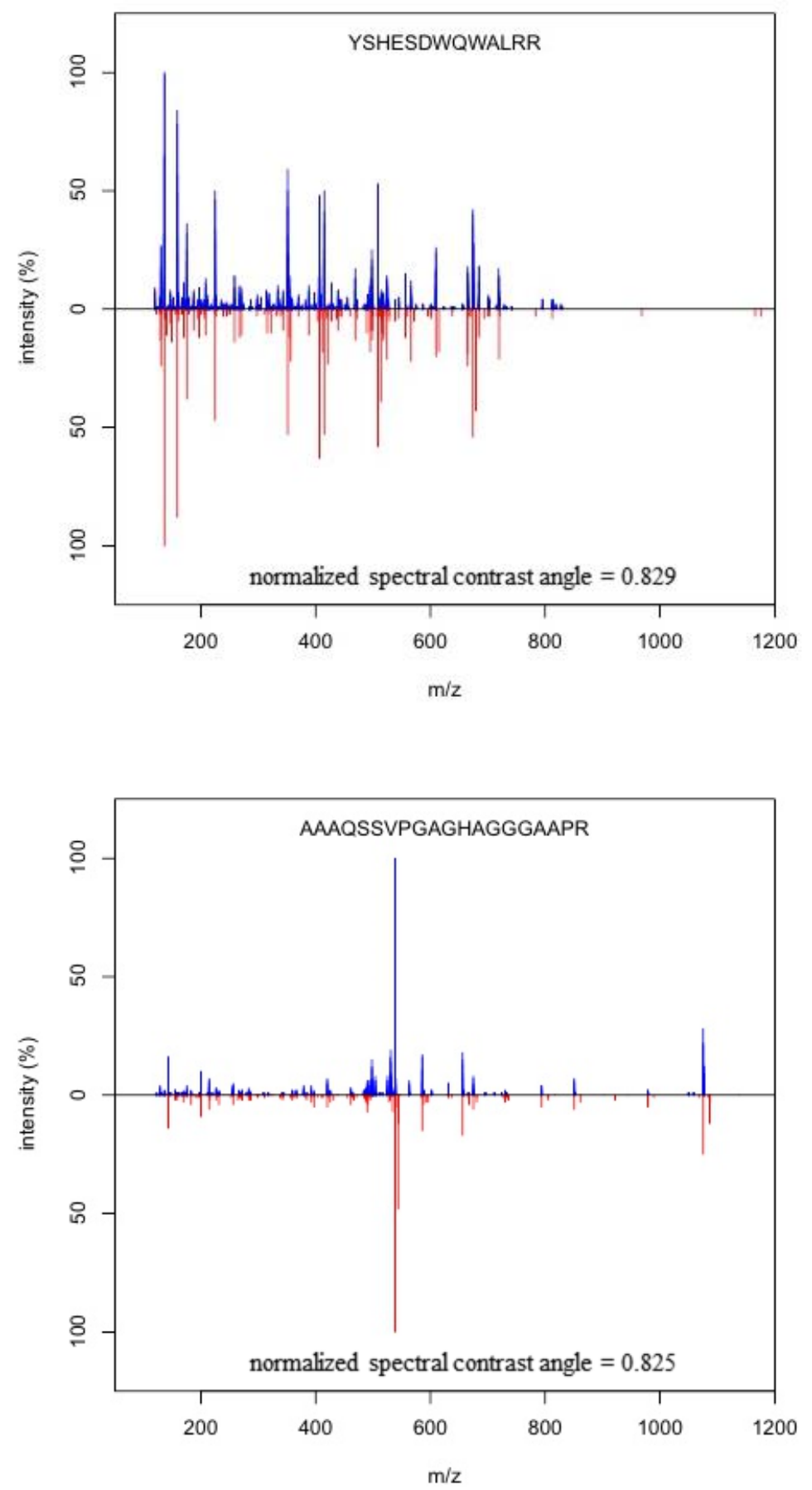

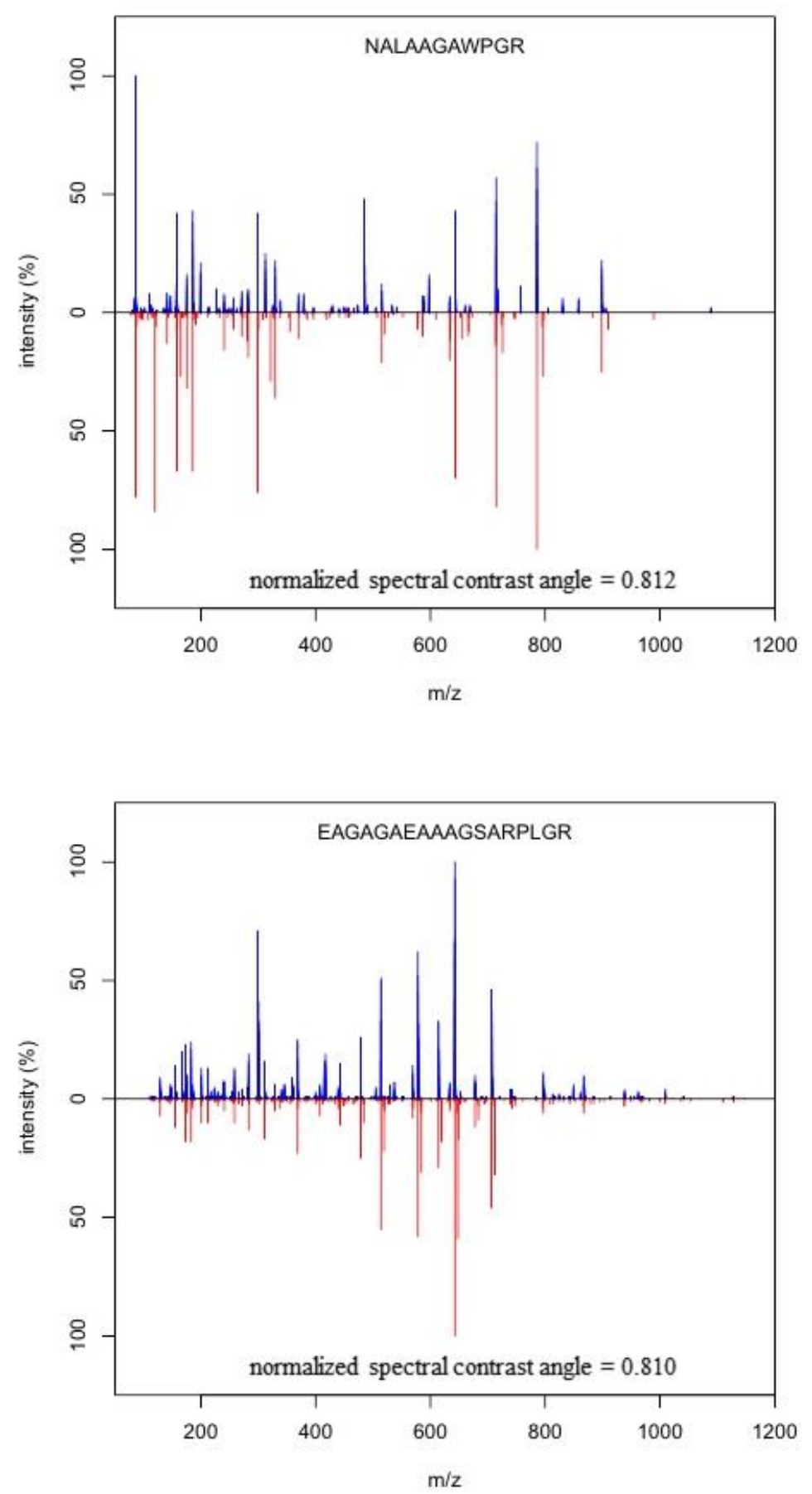

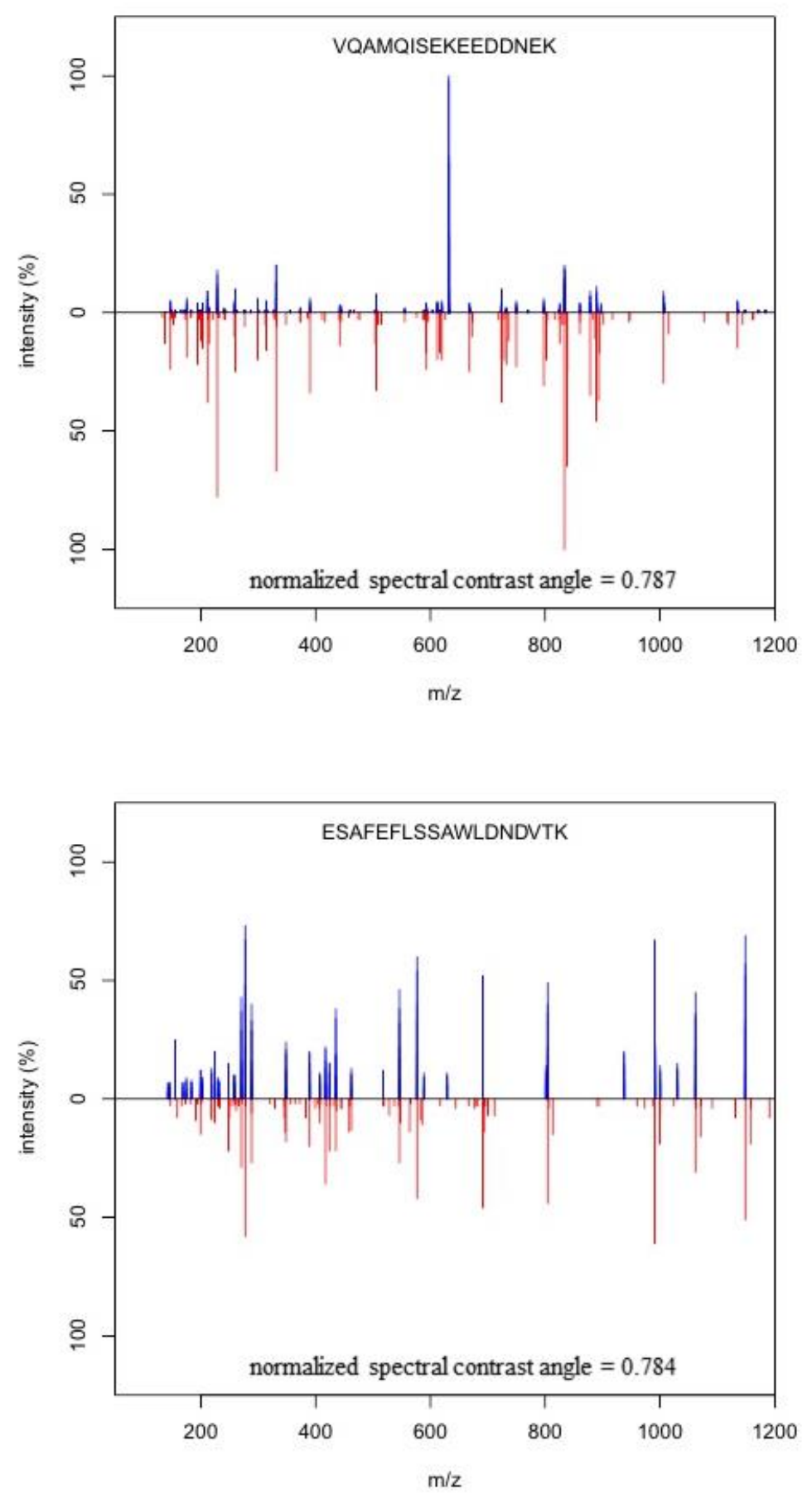

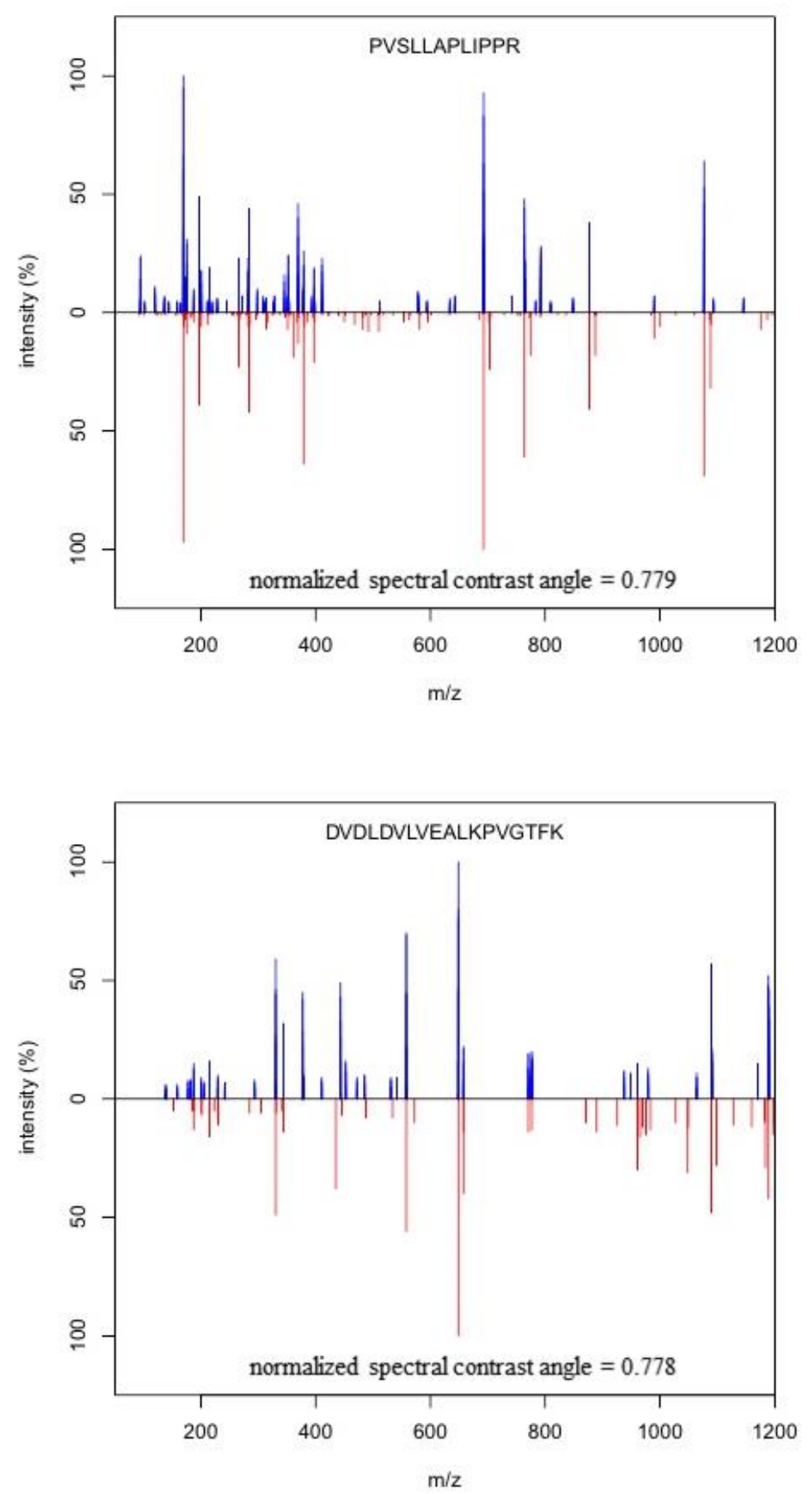

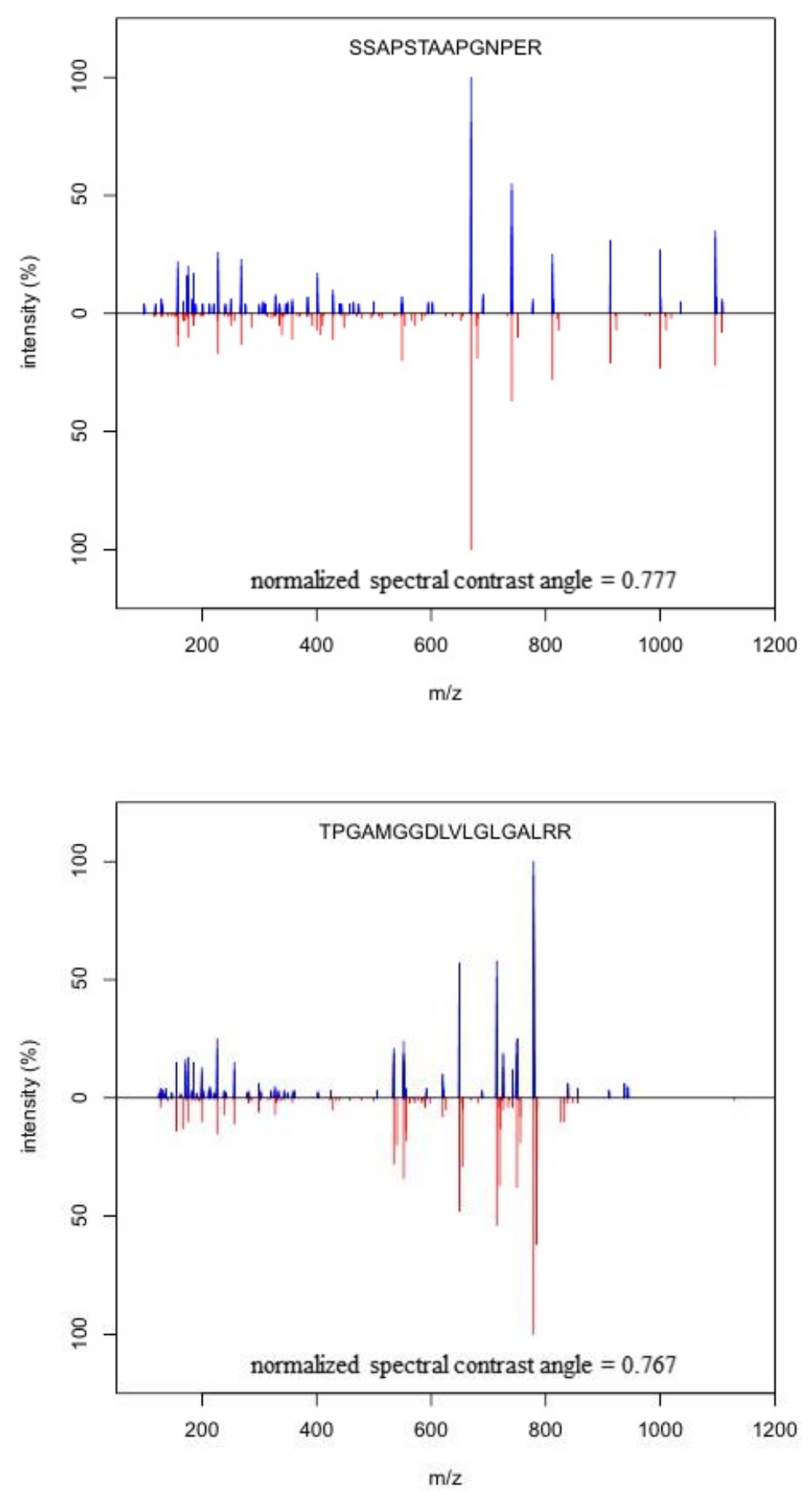

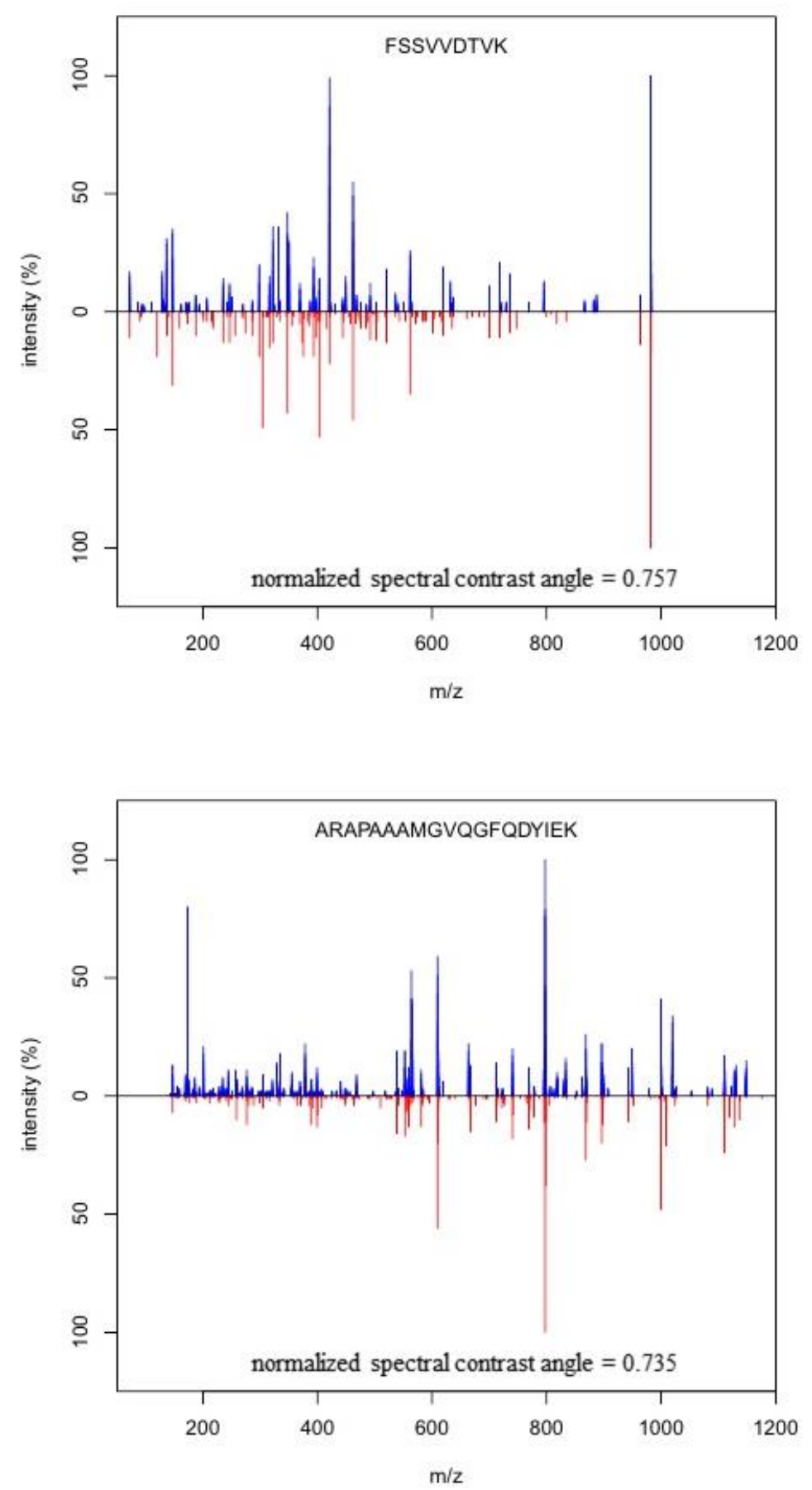

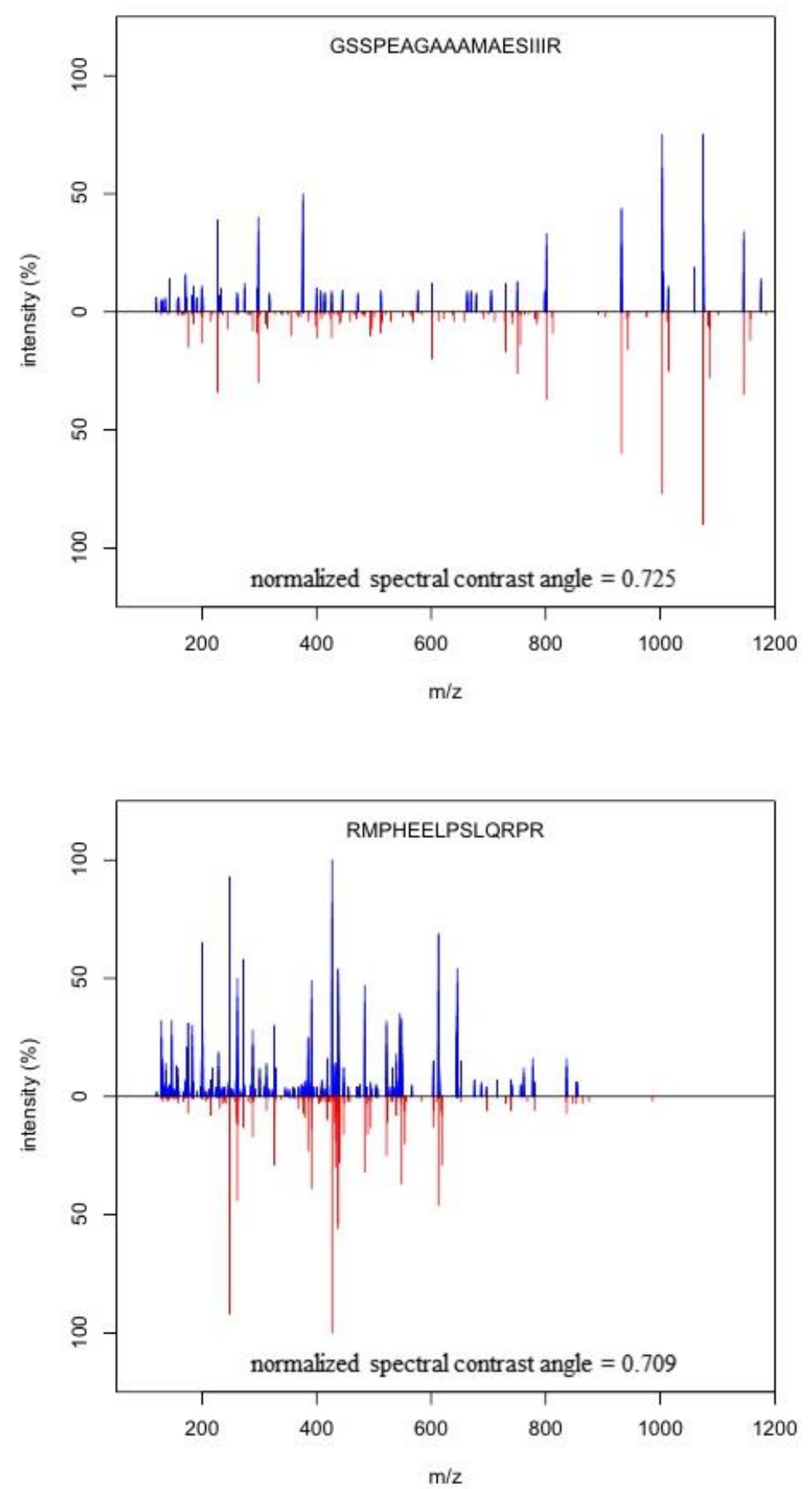

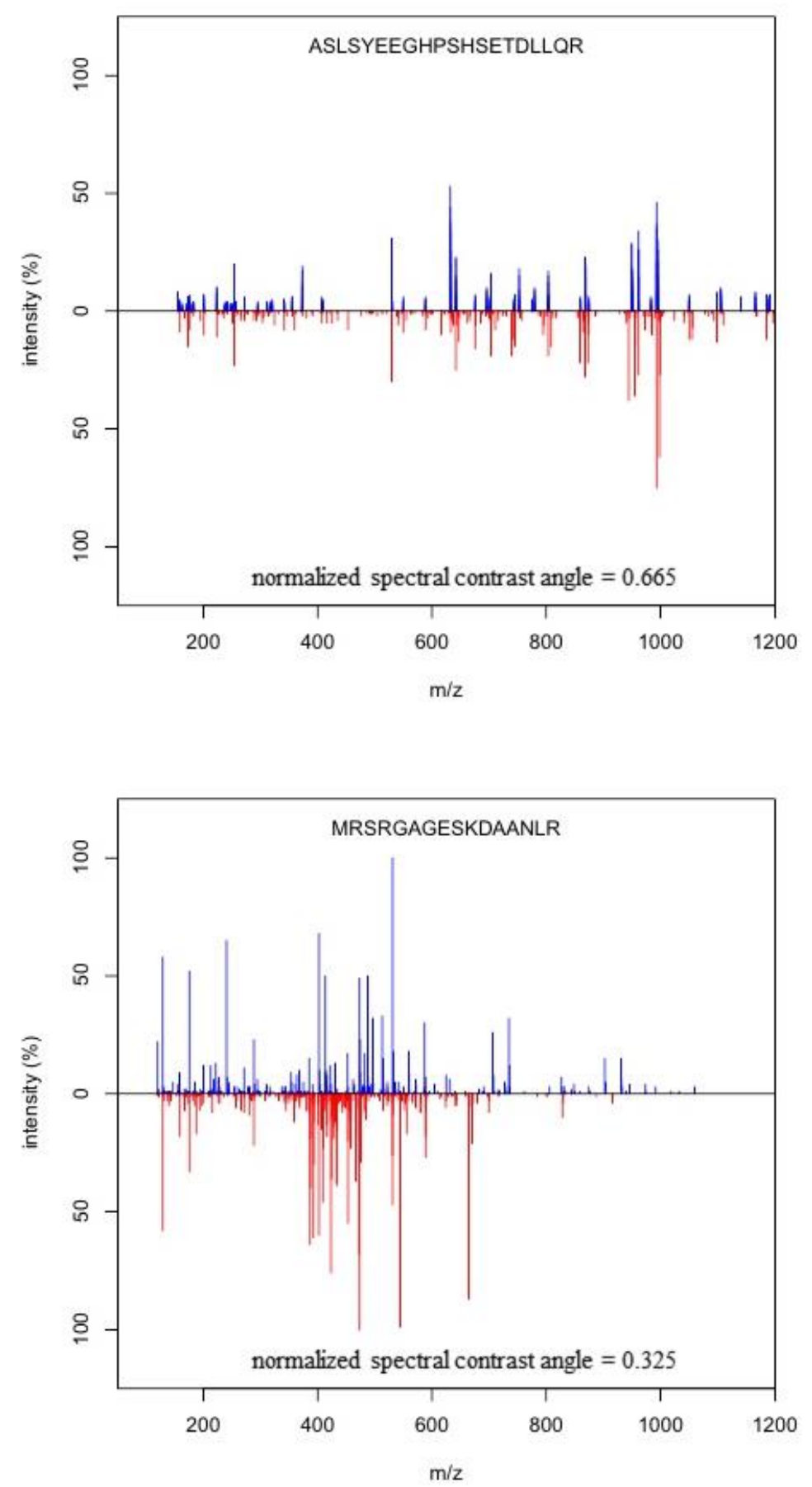